IFBB

NACHWUCHSFORSCHER

ENTWICKELN

NEUARTIGE BIOBASIERTE

VERBUNDWERKSTOFFE

WEITER

Das Institut für Biokunststoffe und Bioverbundwerkstoffe (IfBB) an der Hochschule Hannover erhält bis mindestens 2015 eine der bundesweit sechs neuen Forschernachwuchsgruppen, die vom Bundesministerium für Ernährung, Landwirtschaft und Verbraucherschutz (BMELV) im Themengebiet der stofflichen und energetischen Nutzung von Biomasse gefördert werden.

Ziel der Arbeit der Forschernachwuchsgruppe „Systematische Identifizierung sowie praktische Umsetzung von Synergien im Bereich der Biopolymere, Biopolymerfasern und Verbundwerkstoffe" ist neben der Grundlagenforschung vor allem die anwendungsorientierte Weiterentwicklung und Marktetablierung neuartiger biobasierter Verbundwerkstoffe, beispielsweise für den Automobil- oder Sportartikelbereich. Dazu gehören auch die Optimierung der zugehörigen Prozesstechnik und die Verarbeitung. Herkömmliche Kunststoffe sowie Glas-, Kohle- oder herkömmliche Kunststofffasern als Verstärkungskomponente sollen dabei durch biobasierte Fasern sowie Biokunststoffe oder Bioverbundwerkstoffe nachhaltig substituiert werden.

Die Forschernachwuchsgruppe besteht aus vier bis fünf Wissenschaftlern und mindestens einem technischen Mitarbeiter. Die unter der Projektträgerschaft der Fachagentur Nachwachsende Rohstoffe (FNR) an der Hochschule Hannover geförderte Nachwuchsgruppe wird mit 1,1 Millionen Euro vom BMELV sowie zusätzlich mit rund 0,5 Millionen Euro aus der Industrie gefördert.

PROF. THOMAS GRIES

\title{
Ehrenprofessur der Moscow State University
}

Die Lomonosov Moscow State University (MSU) hat Prof. Thomas Gries, Direktor des Instituts für Textiltechnik (ITA) der RWTH Aachen University, die Ehrenprofessur verliehen. Die Würde wurde ihm am 25. Januar 2013 in Moskau zuteil. „Professor Gries wird aufgrund seiner besonderen wissenschaftlichen Leistung im Bereich der Entwicklung faserbasierter Verbundwerkstoffe geehrt", sagte Prof. Victor Antonovich Sadownitschij, Rektor der MSU. Die Ehrenprofessur wird einmal jährlich an einen verdienten ausländischen Wissenschaftler und einen russischen Staatsbürger vergeben. Als Russin sei dieses Jahr die erste Frau im Weltraum Valentina Tereshkova geehrt worden. Mit der Ehrenprofessur hat die MSU bereits verschiedene Nobel preisträger ausgezeichnet, unter ihnen auch Niels Bohr.

Gries erhält die Ehrenprofessur auch für seinen Erfolg in der Vertiefung der deutsch-russischen Wissenschaftsbeziehungen. Sowohl der Austausch von Studierenden und Wissenschaftler als auch Gastvorlesungen und Forschungskooperationen wurden von ihm initiiert, teilt die RWTH Aachen mit.

Thomas Gries studierte Wirtschaftsingenieur- und Ingenieurwissenschaften und promovierte im Bereich Ingenieurwissenschaften mit Auszeich-

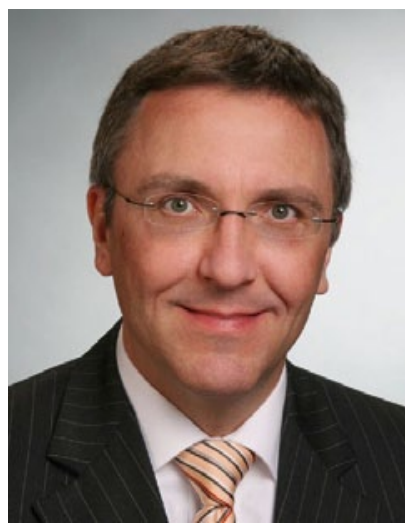

nung. Danach arbeitete er mehrere Jahre in der Industrie in leitender Position. Seit April 2001 ist Gries Institutsleiter am Institut für Textiltechnik (ITA) der RWTH Aachen University mit nunmehr 300 Mitarbeitern, darunter 90 Wissenschaftlern.

Unter der Leitung von Gries wurde am ITA das Konzept "Tailored Reinforcement Textiles" systematisch entwickelt und realisiert von der grundsätzlichen Idee zu einem industriellen Produktionsansatz. Damit wurde die Voraussetzung für die ressourceneffiziente Fertigung von Faserverbundbauteilen und deren breitem Einsatz für Leichtbau-Anwendungen geschaffen.

\section{ÜBERNOMMEN}

\section{Riftec ist Teil der HAl-Gruppe}

Seit Februar 2013 gehört Riftec zur österreichischen HAl-Gruppe. Hammerer Aluminium Industries (HAI) hat zu diesem Stichtag mit europaweit rund 650 Mitarbeitern die Geschäfte des Geesthachter Unternehmens, das im Jahr 2012 in finanzielle Schieflage geraten war, übernommen. Der Name Riftec, der in Fachkreisen eng mit dem Fügeverfahren Rührreibschweißen verbunden ist, bleibt dabei ebenso erhalten wie die wesentliche Struktur des Unternehmens.
Riftec hatte im Spätsommer vergangenen Jahres Insolvenz angemeldet. Nun können die Geschäftsführer, Alexander von Strombeck und Dr. Axel Meyer, nach eigenen Angaben positiv in die Zukunft blicken, denn mit dem österreichischen Privatunternehmen hätten sie einen Investor gefunden, der Riftec gemeinsam mit ihnen zurück auf den Erfolgskurs bringen werde. 